\title{
Electrochemical Sensor for the Controlled-Release of Curcumin from a Novel Polyacrylamide Hydrogel
}

\author{
ShaoPei Li $^{1,2}$, Celia Ferrag ${ }^{1}$, Svetlana Mikhaylichenko ${ }^{1}$, Kagan Kerman ${ }^{1,2 *}$ \\ ${ }^{1}$ Department of Physical and Environmental Science, University of Toronto Scarborough, 1065 \\ Military Trail, Toronto ON, M1C 1A4 \\ ${ }^{2}$ Department of Chemistry, University of Toronto, 80 St. George Street, Toronto, ON, M5S $3 \mathrm{H} 6$ \\ ${ }^{*}$ Corresponding author: kagan.kerman@utoronto.ca
}

\begin{abstract}
:
In this study, we applied an electrochemical detection approach to study the release of curcumin from a polyacrylamide-based hydrogel. Cross-linked polyacrylamide hydrogels have often been utilized for their controlled-release properties. Differential pulse voltammetry (DPV) of curcumin enabled a rapid and costeffective method to determine the release kinetics from the hydrogel matrix with a detection limit of $100 \mathrm{nM}$.
\end{abstract}

Keywords: electrochemical detection, polyacrylamide, high-swelling hydrogel, controlled-release, curcumin

\section{Introduction}

Acrylamide monomers, when polymerized with a cross-linker, such as N,N'-Methylenebisacrylamide (MBA), form high-swelling hydrogels. The structure and size of pores within the polymer can be easily controlled through varying the concentration of crosslinker [1]. Furthermore, due to the three-dimensional network, small molecules can be introduced and trapped inside the matrix. The polymer matrix protects the small molecules from degradation and allows their controlled-release under desired conditions [2].

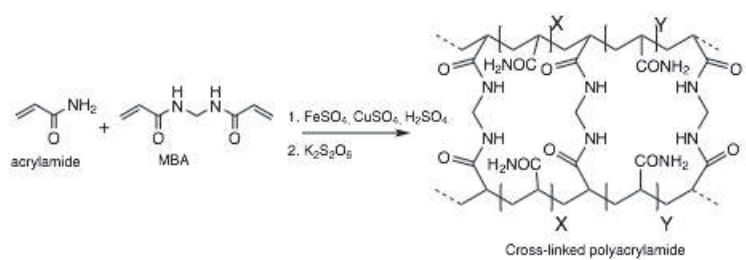

Fig. 1A. Chemical structure of acrylamide, MBA, and cross-linked polyacrylamide (MBA-PAA)

Conventional methods to analyze the controlledrelease processes often involves spectroscopic and chromatographic set up that require sample pretreatment prior to data acquisition [3]. With electrochemistry, however, direct in-situ detection of the compounds released by the hydrogel can be performed in an easy-to-use and cost-effective setup. We have applied differential pulse voltammetry (DPV), which is a highly sensitive and reproducible technique.

In this study, curcumin was chosen as the model compound due to its well-described electrochemical properties [4].

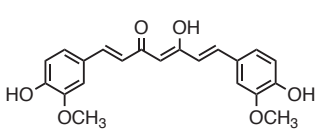

Fig. 1B. Chemical structure of curcumin

Curcumin is a natural colorant compound found in turmeric. Previous studies showed curcumin has positive effects on human health, but due to the chemical structure, curcumin can undergo hydrolysis, protein-induced degradation, and oxidation in the biological systems $[5,6]$. This not only significantly reduces the bioavailability of curcumin, but also, causes individuals to have to consume a large dose of curcumin in order to have a significant impact. Thus, we hypothesized that, if curcumin can be trapped into the hydrogel matrix, and in turn, released into the aqueous environment in a controlled manner.

\section{Methods}

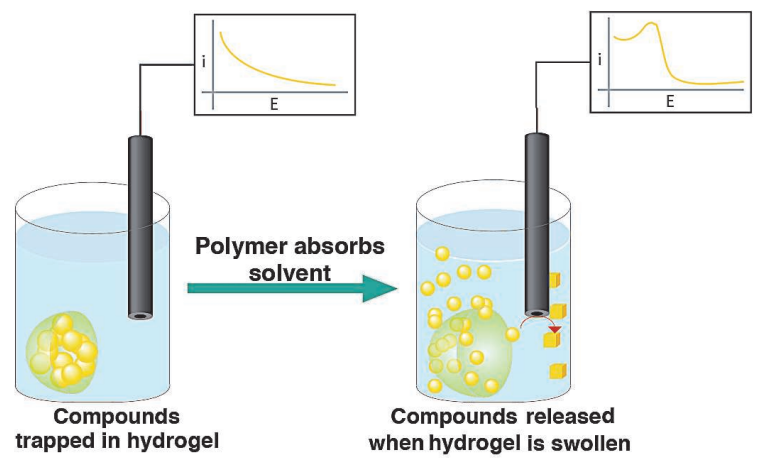

Fig. 3. Experimental set-up of electrochemical detection of curcumin trapped in MBA-PAA polymer.

PAA-MBA hydrogel was synthesized by dissolving acrylamide, $\mathrm{FeSO}_{4} \cdot 7 \mathrm{H}_{2} \mathrm{O}$, and $\mathrm{CuSO}_{4} \cdot 5 \mathrm{H}_{2} \mathrm{O}$ in 
distilled water respectively, under stirring to $50^{\circ} \mathrm{C}$ to achieve a homogenous solution. Then, $\mathrm{H}_{2} \mathrm{SO}_{4}$ was added to acidify the solution. The solution was cooled to room temperature and curcumin was added at a desired concentration. N,N'Methylenebisacrylamide (MBA) was introduced as the cross-linking agent and finally, a saturated $\mathrm{K}_{2} \mathrm{~S}_{2} \mathrm{O}_{8}$ catalytic solution was added to induce polymerization of the hydrogel. The polymer was dried overnight in a $60^{\circ} \mathrm{C}$ oven to remove all the water. Glassy carbon electrodes (GCEs) were polished to a mirror-like finish using 0.3 , and 0.05 micron alumina powder respectively. GCEs were then washed with distilled water and anhydrous ethanol. Three-electrode system was utilized with GCE as the working electrode and a Pt wire as a counter electrode with a $\mathrm{Ag} / \mathrm{AgCl}$ reference electrode Before measurement, the hydrogel was combined with 1xPBS (pH 7.4) and $100 \mathrm{mM} \mathrm{KCl}$. In the defined time increments, clean blank GCEs were exposed to the hydrogels in buffer to perform DPV at a pulse amplitude of $50 \mathrm{mV}$, a pulse width of $0.2 \mathrm{~s}$, and a pulse period of $0.5 \mathrm{~s}$.

\section{Results and Discussion}

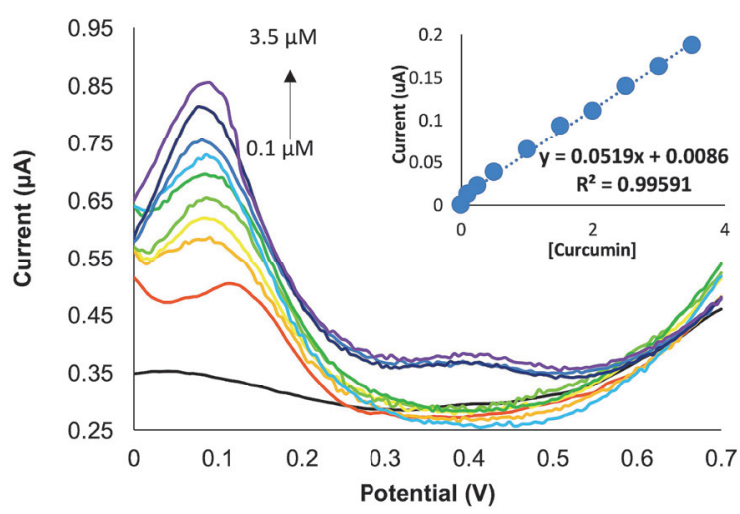

Fig. 4A. Differential pulse voltammograms of curcumin at different concentrations using GCE in $1 \times P B S(p H ~ 7.4)$ with $100 \mathrm{mM} \mathrm{KCl}$ (blank signal in black). The inset shows the calibration curve.

As shown in Fig. 4A, $I_{p a}$ peak correlates to the electrochemical oxidation of curcumin at $0.9 \mathrm{~V}$ (vs. $\mathrm{Ag} / \mathrm{AgCl}$ ), which showed linear relationship with concentration. The limit of detection was determined as $100 \mathrm{nM}$.

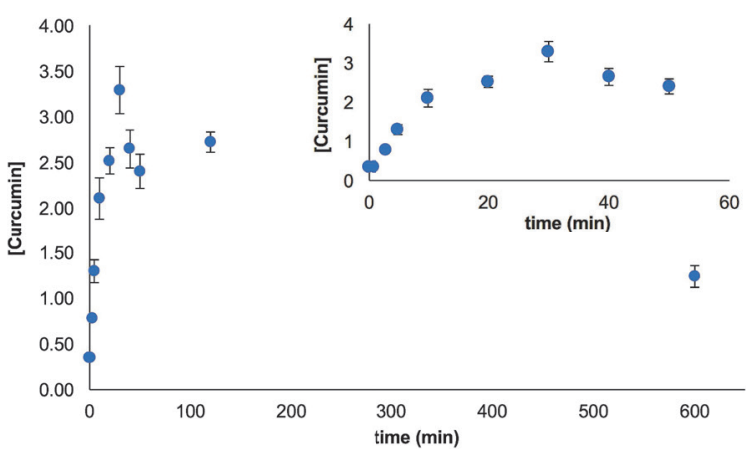

Fig. 4B. Release of $10 \mu M$ curcumin from PAA-MBA polymer in acetonitrile $1 \times$ PBS $(\mathrm{pH}$ 7.4) solution from 0 to $10 \mathrm{~h}$ (inset shows the time range from $0 \mathrm{~min}$ to $60 \min , n=3$ )
As summarized in Fig. 4B, a sharp increase of curcumin release was observed from 0 to $30 \mathrm{~min}$. After $40 \mathrm{~min}$, electrochemical signals slowly decreased. This was attributed to the hydrolysis of curcumin within the aqueous environment. However, the signals remained constant after $24 \mathrm{~h}$. Since the peak potential did not significantly shift, we hypothesized that PAA-MBA hydrogel was able to suppress the degradation of curcumin and sustained the releasing process.

\section{Conclusions}

In this work, a novel PAA-MBA hydrogel was shown to maintain a sustained release of curcumin into the buffer solution. Results indicated PAA-MBA was able to prevent hydrolysis of curcumin prior to release. Our electrochemical approach enabled a rapid and cost-effective chemical sensor to detect and monitor curcumin release process from a novel high-swelling hydrogel.

\section{References}

[1] Ahmed, E. M. Hydrogel: Preparation, Characterization, and Applications: A Review. J. Adv. Res. 2015, pp 105-121. DOI: $10.1016 /$ j.jare.2013.07.006

[2] Sharpe, L. A.; Daily, A. M.; Horava, S. D.; Peppas, N. A. Therapeutic Applications of Hydrogels in Oral Drug Delivery. Expert Opin. Drug Deliv. 2014, 11 (6), 901-915 DOI: 10.1517/17425247.2014.902047.

[3] Kim, Y. J.; Lee, H. J.; Shin, Y. Optimization and Validation of High-Performance Liquid Chromatography Method for Individual Curcuminoids in Turmeric by Heat-Refluxed Extraction. J. Agric. Food Chem. 2013, 61 (46), 10911-10918 DOI: 10.1021/jf402483c.

[4] Li, K.; Li, Y.; Yang, L.; Wang, L.; Ye, B. The Electrochemical Characterization of Curcumin and Its Selective Detection in Curcuma Using a Graphene-Modified Electrode. Anal. Methods 2014, 6 (19), 7801-7808 DOI: 10.1039/C4AY01492H.

[5] Jäger, R.; Lowery, R. P.; Calvanese, A. V; Joy, J. M.; Purpura, M.; Wilson, J. M. Comparative Absorption of Curcumin Formulations. Nutr. J. 2014, 13 (1), 11 DOI: 10.1186/1475-2891-13-11.

[6] Priyadarsini, K. I. The Chemistry of Curcumin: From Extraction to Therapeutic Agent. Molecules. Molecules December 1, 2014, pp 20091-20112. DOI: 10.1039/C4AY01492H 